\title{
Eficiencia mecánica en la soldadura por fricción agitación de la aleación de magnesio AZ31B
}

MATERIALS ENGINEERING

\section{Mechanical efficiency in the friction stir welding of magnesium alloy AZ31B}

\author{
Fernando Franco Arenas*§ \\ *Escuela de Ingeniería de Materiales, Grupo de Materiales Compuestos, Facultad de Ingeniería, \\ Universidad del Valle, Cali, Colombia \\ §ffrancoa@univalle.edu.co
}

(Recibido: Abril 6 de 2010 -Aceptado: Abril 9 de 2012 -Version Final: Mayo 24 de 2012)

\begin{abstract}
Resumen
El propósito del presente trabajo fue determinar la correlación entre las microestructuras y las propiedades mecánicas resultantes en la unión soldada por fricción-agitación de la aleación de magnesio AZ31B, como una función de las variables principales del proceso implementado a partir de una maquina fresadora universal acondicionada. Se lograron soldaduras sanas, sin defectos, para diferentes niveles de velocidad de rotación y velocidad de avance, con valores correspondientes de resistencia mecánica de la unión soldada cercanos a la del metal base, con una eficiencia mecánica máxima del 93\%. La caracterización microestructural mostró granos equiaxiales homogéneos con tamaño promedio de $10 \mu \mathrm{m}$, en la zona agitada. La falla de las probetas durante el ensayo de tensión se presentó generalmente en el lado de avance, con una morfología superficial de tipo dúctil pero con baja deformación plástica aproximada del $5 \%$. Los resultados de calidad y de propiedades mecánicas de las uniones soldadas por fricción-agitación de aleaciones de magnesio AZ31B, permiten asegurar la conveniencia de ser usadas para aplicaciones estructurales teniendo en cuenta su elevada y conveniente relación resistencia mecánica/peso.
\end{abstract}

Palabras Claves: Soldadura por fricción agitación, Aleación de magnesio AZ31B, Eficiencia mecánica, Relación velocidad de rotación/velocidad de soldadura

\begin{abstract}
The aim of the present work was to determine the relationship between the microstructures and the resultant mechanical properties in the Friction stir welded joints of the Mg alloys AZ31B, as a function of the main process variables, implemented from the use of a conditioned universal milling machine. Sound welds without defects, with corresponding high values of mechanical strength, were obtained for different levels of the ratio rotation speed/welding speed of the welded joints, with the maximum mechanical efficiency obtained of $93 \%$. The microstructural characterization in the stirred zone showed fine equiaxed homogeneous grains with an average diameter of $10 \mu \mathrm{m}$. The failure of tension specimens occurred generally trough the advancing side, showing a surface morphology ductile-like with a low plastic deformation of less than 5\%. Quality and mechanical results of FSWed joints of $\mathrm{Mg}$ alloy AZ31B, let us to assure the convenience of using them for structural applications taking into account their high specific mechanical strength.
\end{abstract}

Keywords: Friction stir welding, Magnesium alloy AZ31B, Mechanical efficiency, Rotation peed/welding speed rate 


\section{Introducción}

El proceso Friction Stir Welding, (FSW), traducido como Soldadura por FricciónAgitación, SFA, es una técnica de soldadura en estado sólido, inventado en el Technological Welding Institute, TWI, de Inglaterra en 1991, inicialmente usado para soldar aleaciones de aluminio, permite obtener uniones soldadas de diversos materiales a alta velocidad, sin material de aporte, con propiedades mecánicas y metalúrgicas mejoradas y sin requerir una especial habilidad del operario de soldadura. Se han realizado muchas investigaciones sobre la influencia de las variables más relevantes del proceso como la velocidad de rotación, $\mathrm{V}_{\mathrm{R}}$ [rpm], la velocidad de soldadura, $\mathrm{V}_{\omega}[\mathrm{mm} / \mathrm{min}]$, la presión o carga aplicada y la geometría del pin sobre el tamaño y la penetración de la soldadura y sobre las propiedades mecánicas en relación con las transformaciones de fase en las distintas zonas de la unión soldada de las aleaciones de magnesio. Recientemente se ha enfocado la investigación hacia la búsqueda de nuevas aplicaciones estructurales de las uniones por SFA para la industria del transporte buscando optimizar la resistencia específica y con ello la economía en el consumo de combustible y la reducción de las emisiones contaminantes al medio ambiente.

Johnson (2003a) evaluó el efecto de la relación de velocidad de rotación a velocidad de soldadura, $V_{R}$ $/ \mathrm{V}_{\omega}$, entre 2.2 y $1.1 \mathrm{rev} / \mathrm{mm}$, sobre la SFA de aleaciones de magnesio usadas para fabricar componentes de la industria automotriz y encontró que deben usarse menores velocidades de rotación y mayores alturas del pin de la herramienta, comparada con la soldadura de las aleaciones de aluminio, para reducir el sobrecalentamiento durante la soldadura de las aleaciones de magnesio.

Abbasi et al. (2006), encontró que al aumentar la relación de velocidades $V_{R} / V_{\omega}$, entre 2.5 y 70 $\mathrm{rev} / \mathrm{mm}$, aumenta el calor generado y la agitación del cordón; por lo tanto, aumenta la fricción y el trabajo de deformación plástica produciendo un cordón de soldadura más grande y con menos defectos en la raíz del cordón. Se encontró también que al aumentar la relación $\mathrm{V}_{\mathrm{R}} / \mathrm{V}_{\omega}$ se produjo un leve decrecimiento en la resistencia de fluencia, la resistencia ultima y la elongación de las distintas zonas de la unión soldada.

Xunhong, \& Kuaishe (2006), estudio el efecto de las $V_{R} / V_{\omega}$ entre 6.3 y 25.3 , para las velocidades de rotación 950 y $1500 \mathrm{rpm}$, sobre los cambios de microestructura y propiedades mecánicas de la SFA en aleaciones AZ31 y encontró que la microestructura de la Zona Agitada, ZA, sufría un gran refinamiento de grano, comparados con el metal base ,por efecto de la recristalización dinámica; mientras que en la Zona Termomecánicamente Afectada, ZTMA, los granos son severamente deformados y que presentan una dispersión muy fina de fases intermetálicas del tipo $\mathrm{Mg}_{17} \mathrm{Al}_{12}$.

Esparza et al. (2002), reportó que la ZA presenta granos finos recristalizados con una alta densidad de dislocaciones, y que no hay una diferencia significativa con las otras zonas de la unión soldada, para explicar las superiores propiedades mecánicas obtenidas. Suhuddin et al. también encontraron que la microestructura de la ZA en la SFA de la aleación de Mg AZ31B exhibe granos finos equiaxiales recristalizados. Sin embargo, señalaron que la evolución microestructural durante el proceso SFA de las aleaciones de magnesio no ha sido aun clarificado y los mecanismos reales para la formación de tales microestructuras se mantienen aun confusos. La microestructura en la ZA usualmente no contiene maclas, aunque si revelan una alta densidad de dislocaciones en el interior de los granos.

Darras et al. (2007), usaron una máquina fresadora vertical con control numérico para el procesamiento por fricción-agitación de la aleación comercial de magnesio AZ31B en láminas de 3,2 $\mathrm{mm}$ mostrando un incremento de la dureza y de la resistencia mecánica con el incremento de la velocidad de soldadura, lo cual muestra una buena concordancia con los resultados publicados.

Murillo (2008), implementó el proceso SFA en la Escuela de Ingeniería de Materiales de la Universidad del Valle a partir de una fresadora universal y con esto realizo el estudio de las uniones soldadas de la aleación de aluminio 6261- 
T5 usada para aplicaciones estructurales, obteniendo uniones soldadas sanas con una eficiencia mecánica máxima del $84 \%$. Sánchez \& Betancourt (2008), evaluaron la relación entre la microestructura y las propiedades mecánicas de las uniones por SFA de la aleación de Mg AZ31B usando la fresadora con una herramienta cilíndrica roscada, encontrando un leve incremento de la resistencia a la tracción y la dureza de la unión soldada con el aumento de la velocidad de soldadura, con mejores resultados para las velocidades de rotación mayores. La eficiencia máxima obtenida para una velocidad de rotación de $1800 \mathrm{rpm}$ fue del $85 \%$.

\section{Procedimiento Experimental}

El material usado en el desarrollo de esta investigación corresponde a la aleación de magnesio AZ31B, con una composición nominal de Mg-3Al-1Zn (\% en peso), en forma de láminas con espesor de $3,2 \mathrm{~mm}$ que fueron cortadas de perfiles estructurales extruidos. Se realizaron uniones soldadas a tope sobre láminas de $80 \times 130$ $\mathrm{mm}$, en sentido transversal a la dirección de flujo plástico del perfil extruido, mediante el uso de una máquina fresadora universal de $4 \mathrm{HP}$.

La herramienta de pin cilíndrico roscado fue maquinada en acero $\mathrm{H} 13$ con tratamiento térmico para una dureza mínima de 50HRC, con dimensiones de $12 \mathrm{~mm}$ de diámetro del hombro, 4,5 $\mathrm{mm}$ de diámetro del pin y 2,9 $\mathrm{mm}$ de altura del pin. Durante la soldadura se usó un ángulo de inclinación del cabezal de $1,5^{\circ}$ contrario a la dirección de avance de la soldadura. La Tabla 1 muestra las relaciones de velocidad $V_{R} / V_{\omega}$ para las combinaciones de velocidad de rotación, $V_{R}, y$ velocidad de soldadura, $\mathrm{V}_{\omega}$, usados en la ejecución de las uniones soldadas.

Tabla 1. Relaciones de velocidad de rotación/soldadura para las distintas combinaciones de las condiciones de soldadura definidas en la ejecución de las probetas.

\begin{tabular}{cccc}
\hline $\begin{array}{l}\text { Velocidad de } \\
\text { Rotación }\end{array}$ & \multicolumn{3}{c}{$\begin{array}{c}\text { Velocidad de Soldadura } \\
(\mathrm{mm} / \mathrm{min})\end{array}$} \\
\hline$(\mathrm{rpm})$ & 100 & 200 & 300 \\
1200 & 12 & & 4 \\
1600 & & 8 & 5,3 \\
2000 & 20 & & 6,7 \\
\hline
\end{tabular}

De cada probeta soldada se cortaron muestras para los ensayos metalográficos y mecánicos. La preparación metalográfica se realizó sobre cortes transversales al cordón de soldadura mediante un desbaste inicial con lijas de granulometría decreciente hasta No. 1200, seguido de pulido en paños con alúmina en suspensión de granulometría hasta de $0,05 \mu \mathrm{m}$ y finalmente el ataque químico con el reactivo compuesto por $5 \mathrm{~g}$ de acido pícrico, $10 \mathrm{ml}$ de acido acético, $10 \mathrm{ml}$ de agua destilada y $75 \mathrm{ml}$ de etanol.

El análisis metalográfico se realizó primero con la observación de la macroestructura y geometría de las uniones soldadas en el estéreo-microscopio, seguido del análisis microestructural en cada zona de la unión soldada con un microscopio óptico Olympus LM y finalmente con el microscopio electrónico de barrido JEOL JSM-6490LV, provisto de la unidad de análisis químico por EDS.

Sobre las mismas muestras se realizaron los barridos de microdureza Vickers, con indentaciones cada $1 \mathrm{~mm}$ a través de las zonas de la unión soldada sobre una línea a la altura de la mitad del espesor de las láminas soldadas usando un Microdurómetro Instron-Wilson modelo 401MVD, con carga aplicada de 200g durante un tiempo de $10 \mathrm{~s}$.

Las probetas para los ensayos de tensión se maquinaron de las láminas soldadas, con la dirección de carga paralela a la dirección de flujo plástico del material y transversal al cordón de soldadura. Las dimensiones aproximadas de la sección reducida fueron de $3,2 \times 12,7 \mathrm{~mm}$ con una longitud de prueba de 50,8 $\mathrm{mm}$. Los ensayos de tensión se ejecutaron en la maquina universal de ensayos Instron 3369 con capacidad de 50KN con una velocidad de carga de $1 \mathrm{~mm} / \mathrm{min}$. Las probetas ensayadas se observaron en el microscopio SEM para definir la morfología de la superficie de fractura.

\section{Resultados y discusión}

\subsection{Análisis metalográfico}

La observación de las uniones soldadas en la sección transversal mediante el estéreomicroscopio permite definir los defectos de 
soldadura y la morfología de los cordones de soldadura. Se puede observar que a bajas velocidades de soldadura la calidad de la soldadura es óptima, dado que no presentan defectos apreciables. Por otra parte, a velocidades de soldadura altas se encontraron algunos poros especialmente en el lado de avance que se extienden en una sucesión de poros en la raíz del cordón. La geometría del cordón es aproximadamente cónica con penetración completa hasta la raíz del cordón, como se muestra en la Figura 1.

La microestructura del metal base, mostrada en la Figura 2, está compuesta por grandes granos de tamaño promedio ASTM No. 5,0 (correspondiente a un diámetro aproximado de $65 \mu \mathrm{m})$ y presenta una cantidad significativa de inclusiones no-metálicas alineadas en el sentido de las bandas de deformación del proceso de extrusión, que varían en cantidad y distribución de una muestra a otra. De igual forma, se aprecia claramente la presencia de maclas en el interior de los granos por efecto del proceso de extrusión previo.
La microestructura de la ZA presenta granos equiaxiales finos de un tamaño uniforme promedio ASTM No. 10,5 (correspondiente a un diámetro aproximado de $10 \mu \mathrm{m})$, de igual forma como lo han reportado Suhuddin et al. Se observó que el tamaño de grano aumenta levemente con el incremento de la velocidad de rotación. La distribución y alineación de las inclusiones cambia notoriamente en la ZA, con respecto a la distribución observada en el metal base. No se observaron maclas en el interior de los granos refinados. Ver Figura 3.

En la Zona Termomecánicamente Afectada (ZTMA) los granos presentan morfología y tamaños variables, donde además se aprecian las bandas de flujo plástico del material y la evidencia de una recristalización parcial. Ver Figura 3. La microestructura de la ZA, ampliada con ayuda del SEM, como se observa en la Figura 4 muestra el detalle de los granos finos y bien definidos resultantes de la recristalización dinámica por efecto de la severa deformación plástica en caliente ocurrida durante el proceso. A diferencia

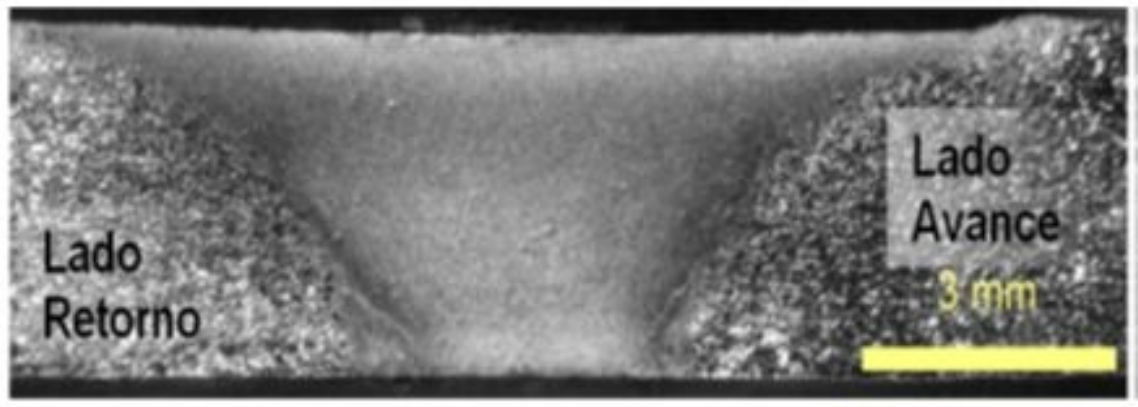

Figura 1. Macroestructura tipica de la union por SFA de la aleación de magnesio AZ31B donde se muestra la forma conica y el ancho promedio de la ZA en el centro sin defectos graves de soldadura.

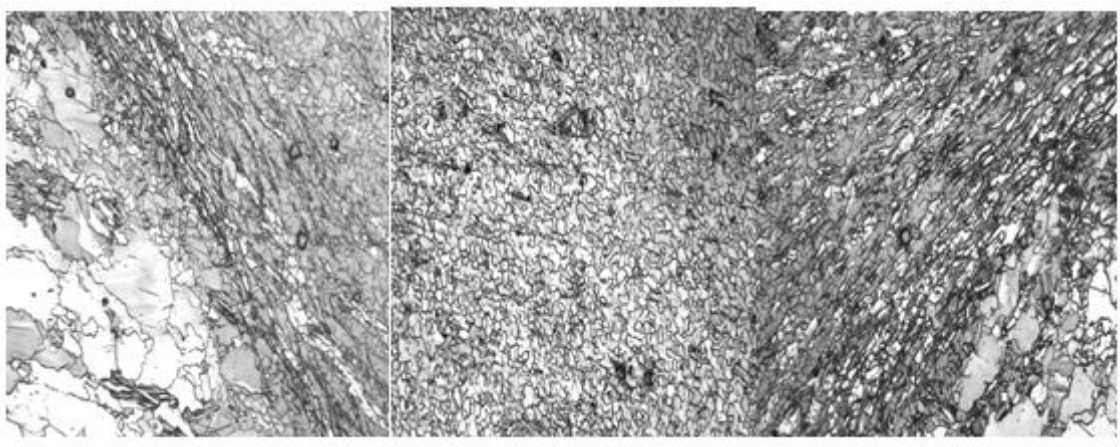

Figura 3. Cambio de microestructura desde la zona de transicion de la ZTMA, con granos heterogeneos en los extremos y la zona agitada de granos equiaxiales finos y uniformes, en el centro. $200 X$. 


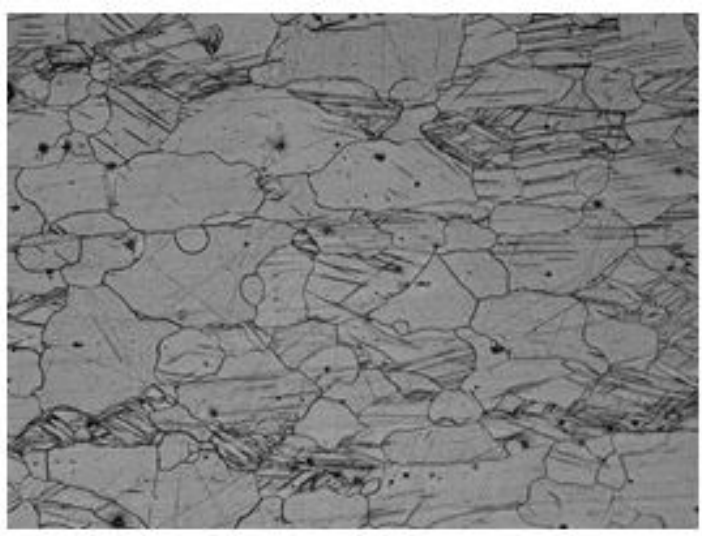

Figura 2. Microestructura tipica del metal base aleacion de magnesio AZ31B conformada por grandes granos, con presencia de múltiples inclusiones no-metálicas y maclas del proceso de conformado en caliente. $100 X$.

de lo reportado por autores como Xunhong \& Kuaishe, (2006), No se encontraron evidencias de la presencia de segundas fases, como la fase intermetálica $\mathrm{Mg}_{17} \mathrm{Al}_{12}$, precipitadas entre los granos sobresaturados de soluto de la ZA. De manera, que puede suponerse que las mejores propiedades mecánicas de la zona agitada debe explicarse por la presencia y el efecto de una alta densidad de dislocaciones al interior de los granos finos, como lo reporta Esparza et al. Las inclusiones no-metálicas, observadas en una proporción apreciable en el metal base de algunas muestras, ver Figura 5a, están compuestas básicamente por óxidos mixtos de Aluminio, Magnesio y Manganeso, como se observa en el espectro EDS mostrado en la Figura 5b.

\subsection{Ensayos mecánicos}

Los resultados de los ensayos de tensión sobre las probetas normalizadas mostraron inicialmente una tendencia de la resistencia a tensión a aumentar con el incremento de la relación de velocidad de rotación/soldadura hasta llegar a un máximo para una relación de 8 , a partir del cual se aprecia una tendencia clara a disminuir con el incremento de la relación de velocidad. Ver Figura 6. Esto puede explicarse por el rol de los defectos de soldadura presentes para altas velocidades de soldadura, pero a velocidades de soldadura

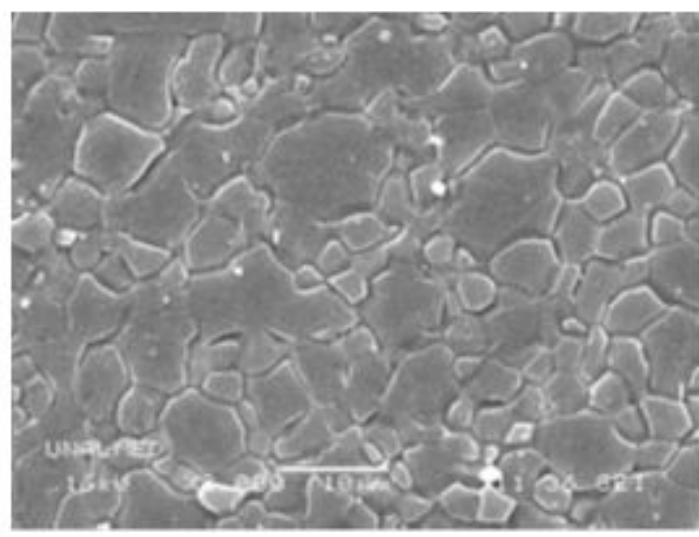

Figura 4. Detalle de los granos finos encontrados por SEM en la zona agitada con un tamaño aproximado de $10 \mathrm{um}$. No se observan precipitados o segundas fases entre los granos de la matrix de la solución solida.

(a)

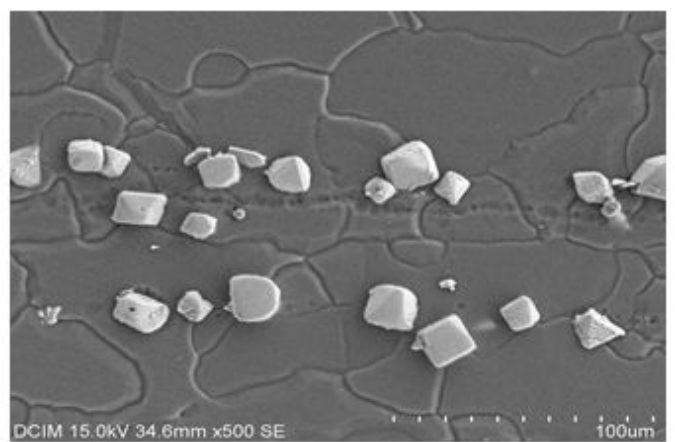

(b)

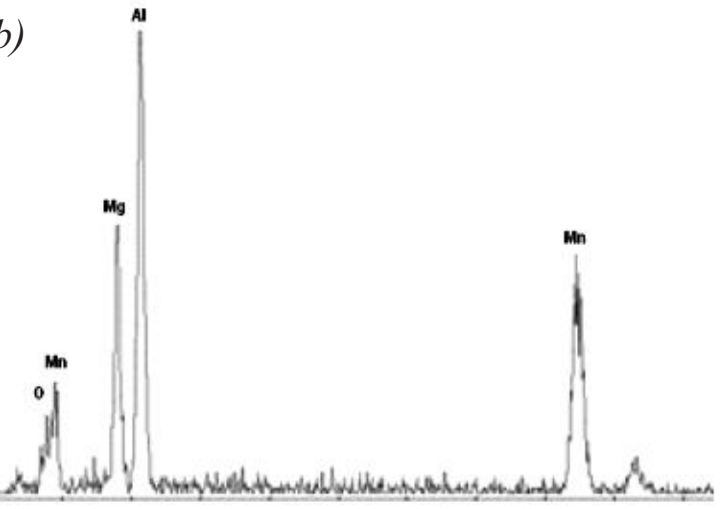

Figura 5. a)Aspecto de las inclusiones no metalicas encontradas en el material base. b)Espectro del Anáisis quimico tipico obtenido muestra que corresponden a óxidos complejos de Mg, Aly Mn. 
intermedias, sin defectos apreciables de soldadura las propiedades mecánicas parecen depender más de la velocidad de rotación y de su relación directa con el calor generado por fricción. La eficiencia mecánica máxima de la junta obtenida fue del 93\% tomada con respecto a la resistencia a tensión promedia del metal base obtenida sobre probetas ensayadas del metal base de $220 \mathrm{Mpa}$.

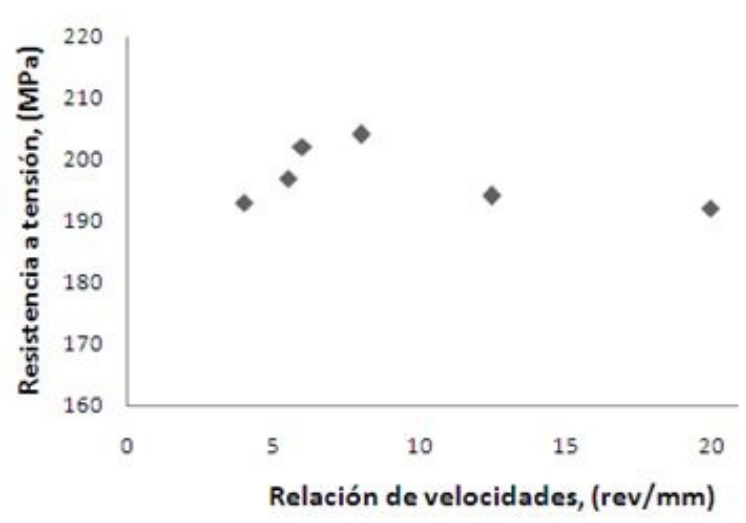

Figura 6. Curva de la Resistencia a la tension de las uniones soldadas en funcion de la relacion de velocidad de rotacion/soldadura.

El plano de falla en las probetas ensayadas se encontró regularmente en la zona de transición o en la ZTMA del lado de avance, con una falla tipo dúctil pero con poca deformación plástica no superior al 5\%. La Figura 7 muestra la ubicación de la falla por la zona de transición sobre un plano a $45^{\circ}$ con respecto a la dirección de aplicación de carga. Se observa además que las ZTMA de lado avance y lado retorno presentan evidencia de deformación plástica comparada con la ZA. La observación de la superficie de fractura en el microscopio electrónico de barrido, SEM, confirma las evidencias de la deformación plástica con crestas y huecos característicos, tal vez relacionados con la posible presencia de inclusiones no metálicas como iniciadores de la falla. Ver Figura 8. Estos resultados nos permiten entender que el material de la zona agitada presenta mayor resistencia mecánica gracias a su microestructura fina, comparada con la estructura compleja de la zona de transición compuesta por bandas de deformación intercalados con granos parcialmente recristalizados.

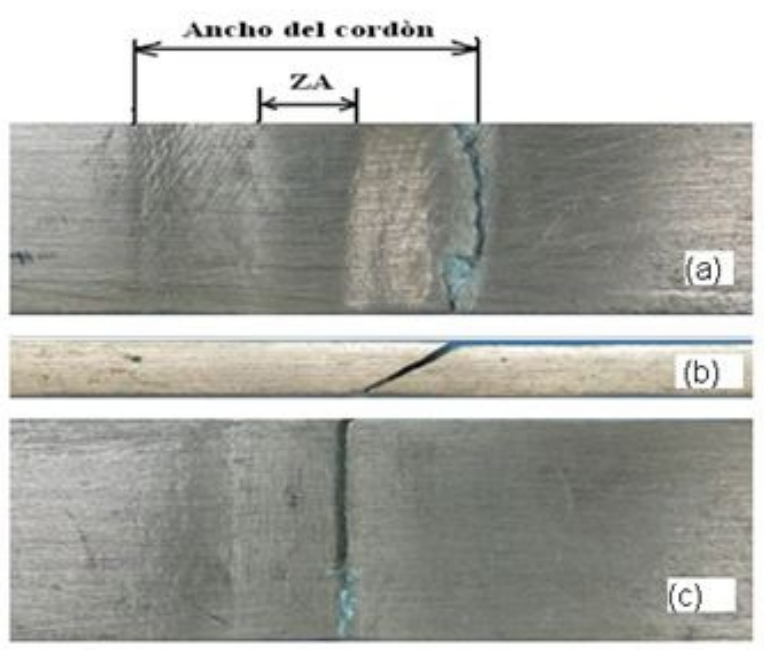

Figura 7. Ubicación de la Superficie de fractura de las probetas ensayadas a tensión por la ZTMA sobre un plano a $45^{\circ}$ respecto a la dirección de carga, vista en a) Superficie de la unión soldada, b) sección transversal y c) cara posterior o raiz de la soldadura.

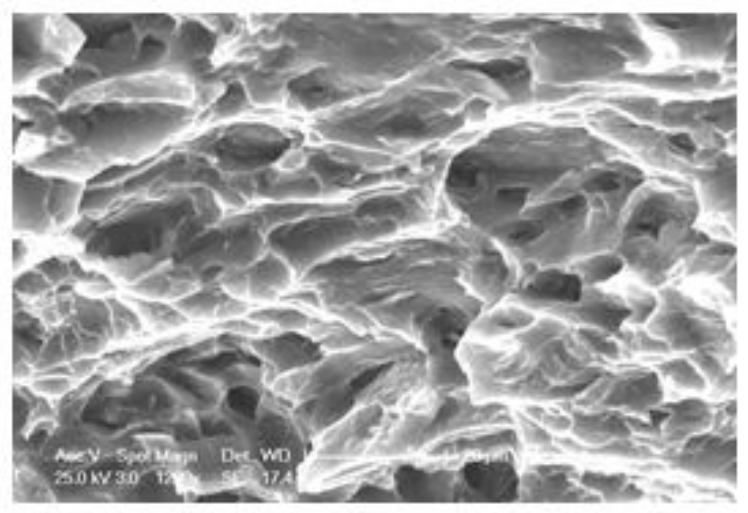

Figura 8. Superficie de fractura característica de las probetas soldadas y ensayadas a tensión con presencia de huecos y crestas observadas por SEM.

Los barridos de microdureza de la Figura 9 no parecen mostrar una tendencia clara a aumentar la dureza en la zona agitada comparada con el metal base con picos alternados al cruzar las zonas del cordón, a diferencia de lo presentado por Darras (10) y Sánchez (11). Sin embargo, aunque las diferencias de microdureza entre los diferentes tratamientos son menores, parece haber un ligero incremento de la dureza en la zona agitada para las probetas soldadas con menor velocidad de rotación. 


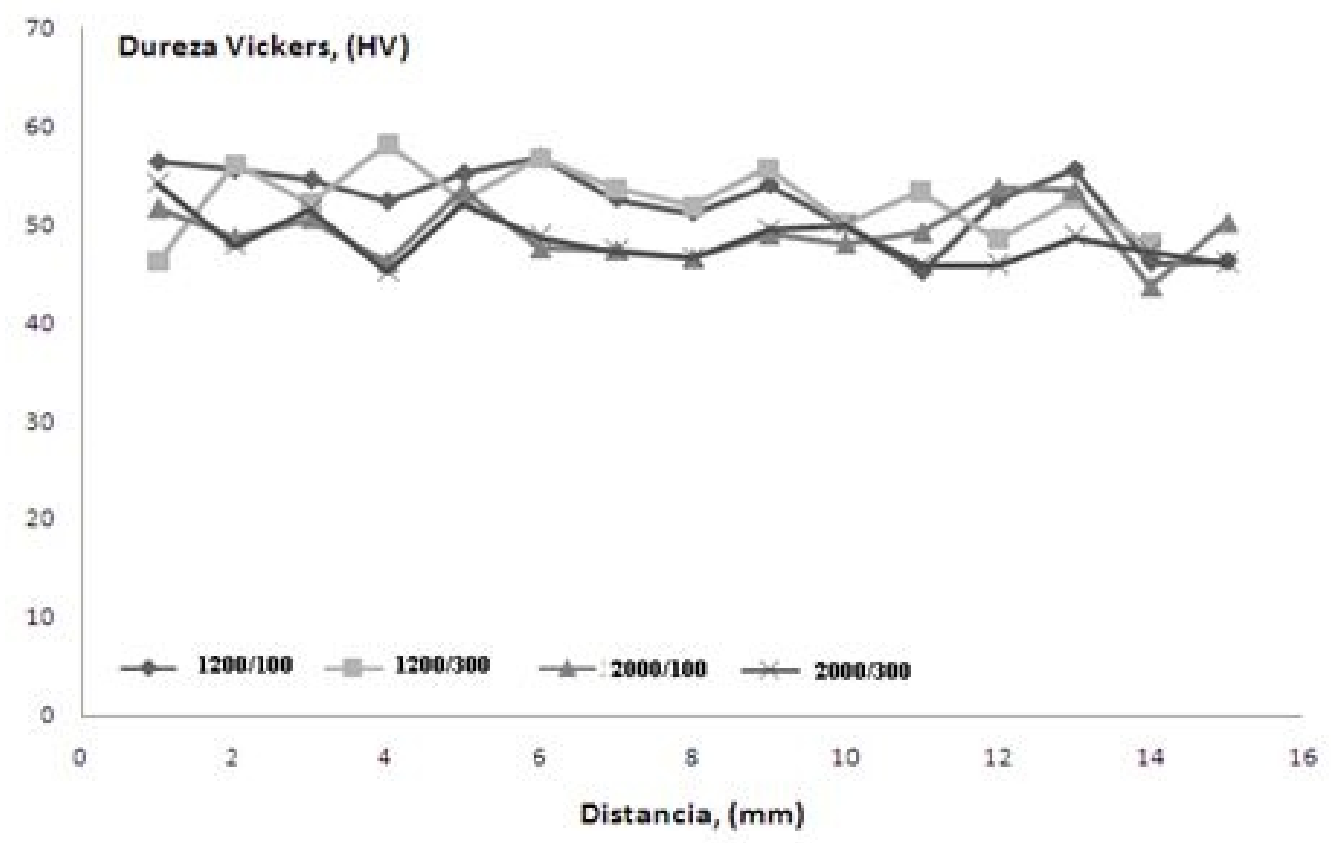

Figura 9. Curvas de microdureza Vickers obtenidas para las diferentes condiciones de soldadura, definidas por la relación de velocidades de rotación/soldadura, como se definió en la Tabla 1.

\section{Conclusiones}

La calidad y sanidad de las uniones soldadas por fricción agitación nos permite asegurar una aplicación confiable del proceso para usos estructurales.

La microestructura general de la zona agitada presenta granos finos uniformes resultantes de la recristalización dinámica que corresponde a una reducción de tamaño de grano superior al $85 \%$ respecto al metal base.

La eficiencia mecánica máxima obtenida en las uniones por FA fue del 93\% que compite con los resultados obtenidos para soldaduras por proceso MIG del mismo material.

Las diferencias menores de microdureza observadas para las diferentes condiciones de soldadura no explican el comportamiento en tensión de las probetas soldadas, razón por la cual debe buscarse la justificación en las transformaciones metalúrgicas ocurridas en las zonas de transición.

\section{Referencias Bibliográficas}

Abbasi, M., Kokabi, A., Daneshi, G., Shalchi, B., Sarrafi, R. (2006). The influence of the ratio of rotational speed/transverse speed on mechanical properties of AZ31B friction stir welds. International Journal of Machine Tools \& Manufacture 46, 1983-1987

Darras, B., Khraisheh, M., Abu-Farha, F., Omar, M. (2007). Friction stir processing of commercial AZ31 magnesium alloy. Journal of Materials Processing Technology 191, 77-81.

Esparza, J., Davies, W., Trillo, E., Murr, I. (2002). FSW of Mg alloy AZ31B. Journal of Materials Science, Letters 21, 917-920.

Johnson, R. (2003). Friction Stir Welding of Magnesium Alloys. Materials Science Forum. 419-422 (1), 365-370. 
Johnson, R. (2003). Further assessment of the FSW of $\mathrm{Mg}$ alloys. The Welding Institute, Electronic copyright 766(3).

Murillo, O. (2008). Desarrollo del proceso de soldadura FSW a partir de una máquina fresadora. Tesis de grado, Ingeniería Mecânica, Universidad del Valle, Cali, Colombia.

Sánchez, H., Betancourt, D. (2008). Evaluación metalúrgica y mecánica de la Soldadura por Fricción Agitación en la aleación AZ31B. Tesis de grado, Ingeniería de Materiales, Universidad del Valle.Cali, Colombia.

Suhuddin, U., Mironov a, S., Sato, Y., Kokawa, H., Lee, C. (2009). Grain structure evolution during friction-stir welding of AZ3 1 magnesium alloy.Acta Materialia 57 5406-5418.

Thomas, W, Nicholas, E., Neddham, J., Murch, M., Templesmith, P., Dawes, C., (1991). Patent Application No. 9125978.8

Thomas, W. (2003). FSW - Recent developments. International Conference on Processing \& Manufacture of advanced materials, Madrid, Spain.

Xunhong, W., Kuaishe, W. (2006). Microstructure and properties of friction stir butt-welded AZ31 Mg alloy. Materials Sci. and Engineering A. 431, 114-117. 